\title{
"Mirror" magnet experiment for quench studies, quench analysis techniques development and in support of superconducting magnet modeling
}

\author{
Stoyan Stoynev (FNAL) \\ November 2020 \\ (edit 15 December 2020)
}

\begin{abstract}
In order to progress on various research points related to superconducting magnets, we suggest performing series of tests with induced and natural quenches on a mirror magnet, probably using a " $11 \mathrm{~T}$ " coil. The "mirror" is to have voltage taps instrumented on half the strands, on both coil ends. It is to be instrumented with multiple spot-heaters, of the order of ten, in designated pole-turn areas. Instrumentation is to include quench antenna array facing the inner coil surface, as well as acoustic sensors on the inner layer pole. Further, acoustic sensors at the magnet ends and an acoustic calibration transducer are to be installed. Thus, we are to test sensitivity and precision of acoustic and quench antenna (QA) array sensors, both at close proximity to the coil, and we are to test and commission acoustic onmagnet real-time "calibration". New or extended DAQ will be tested as well. In addition to diagnostic development, the purpose of the magnet experiment is to investigate current redistribution in the coil, both during quenching and in operating conditions; to assess voltage build up across strands and in the coil segments during quenching, to explore voltage signature differentiation between different type of quenches; to improve modeling of processes during quenching and for magnet design purposes. In this experiment, we expect to be able to characterize the processes during a quench in unprecedented details.
\end{abstract}

\section{Introduction}

When a magnet is tested at FNAL this typically happens as part of a bigger magnet development program or project. In those cases, it is not advisable or possible to take risks that would jeopardize the magnet performance. Thus, we have limitations on what we can do even if tests are considered vital for the development of the field.

Magnet quenching and quench training are linked and not fully understood phenomena although "training" is recognized as a high priority problem to resolve [1]. Causes limiting magnet performance are hard to precisely pin-point case-by-case as too many parameters could be responsible; we can not afford statistical studies on dedicated expensive magnets and repeatability itself is hard to guarantee in such complex structures; to this day we can not predict how a given magnet will perform. Understanding the underlying mechanisms of processes limiting magnet performance requires improvement in instrumentation and DAQ systems allowing for more "diverse" pictures of the phenomena including variety of data types. It is likely that more sophisticated analysis techniques will help as well [2], [3].

This manuscript has been authored by Fermi Research Alliance, LLC under Contract No. DE-AC02-07CH11359 with the U.S. Department of Energy, Office of Science, Office of High Energy Physics. 
Quenches and their possible precursors are complex events that involve electro-mechanical and magnetic changes distributed in space and time, their locality is not always guaranteed. Current redistribution plays an important role in what the signatures of many observed quench signals look like. Although we have good understanding of main characteristics of current redistribution it is still unclear what exactly the role of splices is, leading to competitive assumptions (boundary conditions) [4] needed to analyze magnet data or design magnets. One of the problems is that related tests done so far are mostly on cables alone [4], [5], [6], [7], [8] and not on operating magnets and this is especially true for $\mathrm{Nb}_{3} \mathrm{Sn}$ based data. Studying all aspects of a quench in real magnets is non-trivial and although analysis tools and simulations are ever improving [4], [9], [10], [11], [12], [13], [14], [15] experiments are still unavoidable in probing fine effects during magnet operation and transients.

We are suggesting going forward with a mirror magnet experiment based on existing coil and structure. This minimizes resources for the tests and still allows relevant data taking. The quench phenomenon is to be investigated in controlled environment with plenty of instrumentation. While we are suggesting utilizing a "11 T" mirror magnet for the studies they can be accomplished with other available mirror-structures as well.

\section{Mirror magnet test scope}

\subsection{Diagnostics and instrumentation development}

It is essential that the magnet is carefully instrumented, including with devices that are not "standard" during FNAL magnet tests. Thus, we first describe instrumentation and later we emphasize its connection to phenomena investigation.

All "standard" instrumentation on the magnet is still adequate for the proposed experiment - voltage taps (segments and splices), strain gauges, temperature measurement devices, coil protection heaters. Similar coils from the "11 T" program were tested multiple times in past and there were two mirror magnet tests so far, in particular. The new experiment will explore:

\subsubsection{Spot heater arrays}

Spot heaters will be placed on the inner turn(s) of the coil at distances $2-10 \mathrm{~cm}$. Those may be delivered on flexible traces [16], or be repurposed strain gauges, or other technology previously used. Options are still to be explored in full but FNAL has the experience needed to successfully deploy an array of spot heaters to a coil. At least 16 independent spot heaters can be supported by the test stand. Such an array was never used in a synchronous way so far. We will also investigate the possibility to place a heater on a single strand (see next).

\subsubsection{Voltage taps on strands}

The " 11 T" cable consists of 40 strands and we plan to instrument 20 adjacent strands with voltage taps close to both Nb3Sn-NbTi lead splices. Each voltage tap is to have power and sense channel/wire giving the opportunity to perform 4-wire measurements. The limit of 20 strands comes from channel feed-through limitations. 
We will consider using one additional strand point of contact as a spot heater at a pole turn.

\subsubsection{Acoustic sensors and calibrators}

For the first time we plan mounting acoustic sensors on the pole piece (towards ends) in addition to sensors outside the magnet. The pole sensors will be placed on dedicated grooves ("islands") on the pole or in special mounting points on the pole or eventually on the end parts. This will be decided based on space limitations, experience with both approaches were previously gained with the "15 T" program. Acoustic sensors (with adjacent electronics) were already tested at FNAL.

For the first time we plan to use an acoustic calibration device, ultrasonic transducer, possibly mounted close to the coil on the end parts ("shoes"). The exact position is mainly to be determined by space considerations. If space allows it, the same device will be used to deliver up to $60 \mathrm{~W}$ of ultrasonic power at close proximity to the coil - we have several transducers ranging in maximal power and size to choose from. Along with wave energy flow and interactions across the structure we will have the opportunity to start exploring possible effects of vibrations on friction (for details see [17] and follow references) between coil interfaces by observing changes to quench characteristics.

\subsubsection{Quench antenna}

FNAL is developing multiple quench antenna devices, part of them based on a fairly new QA technology - flexible PCBs (printed circuit boards). The latest developments are foreseen to be available for the mirror magnet experiment and will allow to sense current redistribution locations at centimeter level precision.

\subsubsection{Strain gauges and voltage taps, temperature measurements}

A couple of channels for both strain gauges and regular VT segments will be made available for data taking at higher than $10 \mathrm{kHz}$ rates, likely $100 \mathrm{kHz}$, and during the entire current ramps. Similar readings will be attempted for temperature measurements in the bore close to the coil (a proper device is to be determined). None of those was done so far at FNAL and we will explore the possibility and usefulness of the recorded data.

\subsubsection{Fiber optics}

There is an active fiber optics development at FNAL and this test is a rare opportunity to test it. It may be possible to place a fiber inside the magnet (close to the pole) or it will be on the magnet shell. Data has supplemental value to SG readings and will probably be still in calibration regime.

\subsubsection{DAQ developments}

We will continue improving the DAQ for acoustics already used, establishing this technique as permanent at FNAL. DAQ rates will be $250 \mathrm{kHz}-1 \mathrm{MHz}$, to be decided. DAQ for the VTs and quench antenna will be commissioned in the meantime (electronics already available) and will take data with $100 \mathrm{kHz}$ rate. Both acoustic and QA will take data during most of the current ramps. The same DAQ is a candidate for 
the special high rate $S G$ readings. For low voltage measurements we are to deploy a newly developed DAQ for V-I measurement in this special regime and we may decide to utilize the "spike measurements" system we have for parallel measurements.

\subsection{Phenomena investigation}

Quenches are so far unavoidable part of the life cycle of low-temperature superconducting magnets. Only a R\&D magnet could afford to have excess diagnostic tools and even there we can not completely characterize every quench. Did it occur due to a mechanical or other "event", what energy was released? Or did the critical surface was reached gradually during ramp up? Are there "precursors" to quenches and what type, how do we best sense them, what "prediction power" do they have? Was a given quench initiated at a single "point" (minimum propagation zone), can we determine that for every quench? Can quench observables reveal the quench source underlying physics? Questions can continue. The important point is that we don't know many of the answers because we never tried to draw the full experimental picture in the first place. The measurement dimensions we are thinking about are not so many though they can be extended - voltage, electro-magnetic behavior, mechanics (acoustics), stress/strain, temperature. Ideally, we want to know any of those in the 3D space of the magnet through the entire ramp to quench and beyond. In practice we have limited coverage, and it is worth noting that typically not a single "measurement dimension" is covered in full with an adequate resolution to answer core questions. While it will probably remain for a long time impractical to measure all we want, even if technologically possible, we are far away from optimal coverage for a quench description. It is the question of optimization that we want to address although this often simply means extending our diagnostic capabilities. In any case, the main effort is to take a picture (or movie) of what is happening before and during a quench from multi-physics point of view.

We will have detailed records of training quenches and, likely, ramp/temperature dependence quenches. Then we are to perform tests with single spot heaters as well as an array of spot heaters for targeted quench investigation. All types of measurements will be done for both natural and induced quenches.

\subsubsection{Quench location and development}

Our main quench characterization so far is based on voltage segments in a coil. They are limited number and do not guarantee good spatial resolution of quench location or propagation velocity. The technology is considered "invasive" and can not be used as a standard for fine spacing in coils. On the other hand, at FNAL we take data at $8 \mathrm{kHz}$ plus minus one second around quench detection. This data rate may be inadequate for "precursors" and thus we don't necessarily catch the start of the quench-event span. Indeed, we don't see precursors in voltages though those were often reported elsewhere [18] using high-rate DAQ. Technically, such precursor measurements can be related to "voltage spike" measurements we often take, we will test monitoring a dedicated segment at higher frequency rate in parallel to "regular" data taking. Moreover, data from individual strands will be available at high rate. We expect to gain from a better picture in fast transients of the initial voltage development. 
Acoustics measurements we took so far were able to locate mechanical events in the magnet with about $10-20 \mathrm{~cm}$ longitudinal resolution at best. Sensors were outside of the magnet and wave paths to the sensors go through multiple interfaces. Putting sensors very close to where most of quenches occur, near the pole, is expected to be beneficial and we may be able to determine mechanical and quench events with centimeter precision. This is still to be demonstrated but the main reason for positive expectations come from the increase of the signal-to-noise ratio of close-to-quench sensors. So far we never managed to get useful information from relative or absolute amplitude measurements due to lack of calibration and certainty of stable sensor response. This time we are to get valuable constantly calibrated data and this by itself will serve as an important diagnostic tool. Quench dependencies on spot heater energy and power will be of interest. As presented in [19] acoustic sensors can provide relevant information, but many promising techniques are still in development stage and routines for optimal results are not yet established.

QA developments at Fermilab go in the direction of covering the full innermost layer of a coil (so far). With recent improvements of our devices, we expect good spatial and temporal resolutions, $\mathrm{cm}$ and sub-ms, respectively. This will complete the most important tri-state picture of voltage-acoustic-electromagnetic signatures during and before quench. We will have the full information in the right spots for spotheater quenches and we expect to be able to use the abundant information on natural quenches of the magnet to characterize much better than now the physical processes during and before quench. The proper QA design configurations will be available and at least partially tested on magnets before the proposed magnet experiment. Earlier unpublished tests indicated different quench propagation velocities based on voltage and QA data. While there were multiple imperfections with the previous tests, including lack of full coverage, it is in the plan now to investigate consistency of quench propagation velocities measured by all different type of distributed sensors.

Some temperature sensors and strain gauges close to the pole will be read at $\sim 100 \mathrm{kHz}$ rates and data correlated with the other high frequency DAQ measurements. Those are expected to be complimentary for the time being, but we will be exploring their future use for quench characterization.

The key aspects of the experiment include: $\mathrm{cm}$ and/or sub-ms resolution for all types of data taking, strand level sensitivity for some measurements, natural and "configurable" induced quenches in the same test conditions. Those should allow us to locate the quench source in space and time precisely for each possible quench in the inner layer and there is no accelerator magnet (coil) test we know of that could do that so far. Quench time differences between different data types (when sensitive) will further provide important input for interpretation.

\subsubsection{Quench source}

The origin of a quench is a difficult subject and our aim is to shed more light on it by investigating in details quench signatures. Electro-magnetic data (usually from QA) were successfully [20] but more often coarsely correlated with voltage segment data or coil geometry [21],[22], [23] and the same can be said about acoustic data [19], [24]. Training quenches are thought to be related to sudden energy releases in/by the conductor and it is reasonable to expect that electro-magnetic or 
mechanical (acoustic) data will always sense it - we do expect the source to be of electromagnetic or mechanical nature [25], [26], [27], [28]. Quenches at plateau could be caused by steady approach to the critical surface and data could be more "silent". Comparisons of quenches in both domains will be conducted and confronted with induced quench signatures. Moreover, the data will allow us to eventually distinguish simultaneous closely separated quenches in a coil. In any case, multi-source initiation will be investigated in a controlled environment with multi-spot heaters and data compared with natural quenches.

It was shown [29] that many quenches are likely caused through the slip-stick mechanism. This implies the quenching zone could be either much larger than the minimum propagation zone (MPZ) or there could be multiple semi-simultaneous MPZ. This is the case because energy is released over much larger area (on the coil) and over longer time than in point-like energy releases like epoxy cracking. Moreover, pre-heated conductor (due to fast sliding over interfaces) will show higher voltage rise differential once a single quench develops. With respect to "point energy release" quenches "slip-stick" type quenches are expected to have a) a different coil segment voltage signature; b) abnormal electromagnetic and/or acoustic signals either inconsistent with a single source or showing abnormal quench propagation. We are to investigate those by the spot-heater array, with the aim to characterize and describe fully how to discriminate between such "area" quenches and "point energy release" quenches. Spot heater arrays will help to create both proper distribution of quench sources as well as "pre-heat" areas of interest allowing good control of quench conditions over large, with respect to MPZ, area.

Additional information from temperature and strain sensors as well as single strand voltages will be used to extract more insights for revealing quench sources.

Past dedicated experiments on $\mathrm{Nb}_{3} \mathrm{Sn}$ conductor [30], [31] showed how well we know the main processes describing quench developments in a (test) coil. However, results are typically considered at a large time scale, order of $10 \mathrm{~ms}$ and above, and in mostly simplified conditions. Then we have remarkably good modeling of many processes. Results are not directly linked or linkable to quench sources though. On a small time-scale which is more relevant for fundamental research, detailed models provide a good basis for process description, even on a grand magnet scale [4], [11], [33]. While available software proves to be invaluable for magnet design and performance analysis, we still need to try distinguishing quench cases where multi-physics software can be tested in more complex conditions. Identifying quench types, likely relatable to quench sources, is one of the goals of the currently proposed experiment.

\subsubsection{Current redistribution}

Current redistribution is a phenomenon that plays an important role in magnet stability and performance. It also takes place during quenching and can serve as an indicator of it and possibly as a precursor of it. While the physics of current redistribution is clear and various related processes studied [4[, [5], [12], [32], [33], [34] a significant complication for interpretation in real magnets is the existence of splices (review on splice/joint technology for various superconductors can be found in [35]). Lead splices can be considered in approximation of equal potential for all 
strands or equal current in all strands with the two giving very different current/power loss distribution outcomes [4]. As discussed in the same quoted paper there are no measurements so far supporting any of the two cases in magnets and this makes magnet design and analysis tools less definitive. It is plausible real situations fall in between the ideal ones.

Our proposed experiment have multiple goals in that regard. Measuring changes in (integral) strand contact resistances for the whole coil from room temperature to super-fluid He temperatures is one of them. Those measurements will be used to understand better the resistance matrix developing in the magnet conductor during cool-down, and observe asymmetries developing between strands (if any). It may be possible to relate coil weaknesses to room-temperature data which is always useful. Results will be obtained by "4-wire" resistance measurements between different strand ends. Half the strands can be measured which will give good statistics and approximation for the whole cable. In addition, energy loss measurements will be performed by the electrical method [36] at various ramp rates. Contact resistances and AC losses extracted for the same magnet (coil) will have a significant value for improving magnet modeling [4]. Then resistances themselves will be analyzed in light of previous cable experiments [5], [37], [38].

Quench antennas with centimeter detection precision covering the whole coils will be able to register changes in strand currents at local level. We'll investigate both multi-strand and single-strand spot-heater responses comparing to real quenches. Current redistribution models will be built and validated in those controlled situations, precision well below the twist pitch length will be achieved. The QA will be able to resolve current redistribution variations along the straight section of the cable pole turn during cyclic powering of the magnet at high ramp rates.

Additional small quench antennas will be placed close to the $\mathrm{Nb}_{3} \mathrm{Sn}-\mathrm{NbTi}$ splices, possibly under pressure at the mid-plane. They will be monitoring current redistributions in the splice area and are expected to provide valuable data for interpretation of observations with the rest of the instrumentation. Those antennas were successfully tested up to $250 \mathrm{MPa}$ transverse pressure at room temperatures.

\section{Experiment preparations}

\subsection{Cable tests}

The experiment proposed relies partially on instrumenting individual strands with voltage taps. This was successfully done at Fermilab in past [32] and the technology is also implemented elsewhere [6], [7], [8]. However, the configuration of the voltage taps requires access to the narrow cable side instrumenting many strands at close proximity. Thus, we plan to test it on cable pieces running current through strands in LHe. This should give us confidence the procedure can be successfully repeated on an impregnated coil. Alternatively, we may have to expose an area $(\sim 1 "$ long) on the wide cable side, close to the leads, in which case we have to make changes to the profile of the facing mirror-block, assess and secure structural integrity.

\subsection{Instrumentation set up}


Except for voltage taps on strands the rest of instrumentation is not regarded risky. Spot heaters may require extended work although we are considering printing heaters on flexible printed boards which will reduce technical labor. The number of voltage tap and spot heater channels is determined by limitations in the feed-through channels of the test stand. Instrumenting half of adjacent strands in the cable with two wires each on both ends will give us the necessary for analysis information. Spot heaters will be distributed around the pole at a distance of $2-10 \mathrm{~cm}$ and we plan to have at least 12 of them covering parts of the straight and turn sections well instrumented with magnet voltage taps. Quench antennas will be placed on a support attached to the mirror block facing the coil and also at coil splices. Depending on QA development we will decide which of the QA PCBs we are to deploy. Acoustic sensors/transducers on coil elements (but not the conductor) will facilitate this kind of data taking. Strain gauges will follow standard for mirror magnets patterns (coil/bullets/skin gauges). Fiber optics will be run in testing mode to assess functionality and it will depend on developments before the start of the magnet assembly.

DAQ systems with rates up to $1 \mathrm{MHz}$ will be used for recording most of the VTs, QA and acoustic data. Systems with sufficient number of channels are already available or ready to become available. Special DAQ for nano-volt measurements is also available.

\subsection{Magnet assembly}

The mirror magnet assembly will follow established procedures from the " 11 T" program - there were two mirror magnets tested [39], [40] and currently another one is in preparation for assembly. The coil inside the magnet under preparation is to be the one we plan to experiment on in this proposal. It should be noted that other coils and structures are available to perform the same experiment. The most nontrivial part of magnet preparation will be instrumenting the coil and routing all wires properly before closing the magnet structure. We will go through the usual readiness reviews to assure compliance with established procedures and if necessary, revise planning accordingly.

\subsection{Simulations}

Fermilab experts have know-how on variety of "standard" magnet simulation software although we may need a better suited one for quench studies [4], [11]. Regardless of how much we can extend our expertise we ourselves are to test available models or we will initiate efforts to collaborate on specific parts of the data analysis with developers [4], [11]. In the preparation phase, we will review our options and start working on modeling for the concrete experiment. In any case the experimental data we plan to publish will be invaluable for improving simulations in real magnet operating conditions and magnet design.

\section{Summary}

The proposed experiment will allow us to develop reliable instrumentation and techniques for future tests, including integrated (multi-physics) data-analysis. It 
will help with quench development and current redistribution modeling. With the detailed data in hand, including data taken in well controlled conditions with spotheater arrays, we expect to gain much better understanding of the processes during a quench, and have complimentary techniques of characterizing the quench. This information is vital for understanding and avoiding magnet performance limitations and in trying to prevent training quenches of happening at all.

\section{References}

[1] S. O. Prestemon et al., "The U.S. Magnet Development Program Plan”, 2016, https://www2.lbl.gov/LBLPrograms/atap/MagnetDevelopmentProgramPlan.pdf, accessed 11/19/2020.

[2] D. Hoang et al., "Machine learning for quench prediction", ASC 2020, submitted for publication.

[3] M. Wielgosz, et al., Using lstm recurrent neural networks for monitoring the lhc super-conducting magnets, Nucl. Instrum. Meth. A867 (2017) 40-50.

[4] L. Bottura, M. Breschi, and A. Musso, "Calculation of interstrand coupling losses in superconducting Rutherford cables with a continuum model", Cryogenics 96, 44, 2018.

[5] G. Willering, "Stability of Superconducting Rutherford Cables for Accelerator Magnets", PhD thesis University of Twente, The Netherlands, 2009.

[6] G. Willering et al., "Current redistribution around the superconducting-tonormal transition in superconducting Nb-Ti Rutherford cables" J. Phys.: Conf. Ser. $97012119,2008$.

[7] A. Cubero et al., "Quench dynamics in MgB2 Rutherford cables" , Supercond. Sci. Technol. 31, 045009, 2018.

[8] W. M. de Rapper, "Thermal stability of Nb3Sn Rutherford cables for accelerator magnets", PhD thesis : Universiteit Twente, 2014. $226 \mathrm{p}$.

[9] G. B. J. Mulder, V. N. Tsikhon, and V. S. Vysotsky, "Quench development in superconducting cable having insulated strands with high resistive matrix-Part 2: Analysis", IEEE Trans. Magn., vol. 28, no. 1, pp. 739-742,Jan. 1992.

[10] M. Breschi et al., "Minimum quench energy and early quench development in NbTi superconducting strands", IEEE Trans. Appl. Supercond. 17 2702-5, 2007.

[11] L. Bottura, C. Rosso, and M. Breschi, "A general model for thermal, hydraulic and electric analysis of superconducting cables", Cryogenics, vol. 40, no. 8-10, pp. 617-626, Aug.-0ct. 2000.

L. Bottura, CryoSoft code THEA v. 2.2; 2013.

[12] Bermudez, S. \& Bajas, H. \& Bottura, L.. (2015). "Quench Modeling in High-field Nb3Sn Accelerator Magnets", Physics Procedia. 67. 840-846. 10.1016/j.phpro.2015.06.141.

[13] A. Protopopov, "Simplified Analytical Formulas of Quench Dynamics," in IEEE Transactions on Applied Superconductivity, vol. 25, no. 2, pp. 1-5, April 2015, Art no. 4700205, doi: 10.1109/TASC.2014.2368142.

[14] D. Paudel, "Quench Simulation of Superconducting Magnets with Commercial 
Multi-Physics Software", thesis, CERN-THESIS-2015-090.

[15] Wake, M. et al. "Complete Quench Simulation of Large Solenoid Magnet." IEEE Transactions on Applied Superconductivity 22 (2012): 4704504-4704504.

[16] https://ohmega.com/wp-content/uploads/OhmegaFlex-Brochure.pdf, accessed $11 / 20 / 2020$

[17] M. Popov, "The Influence of Vibration on Friction: A Contact-Mechanical Perspective", Frontiers in Mechanical Engineering. 6. 10.3389/fmech.2020.00069 (2020).

J. Abdo and M. Tahat, "The Effect of Frequency and Amplitude of Vibration on the Coefficient of Friction for Metals", WSEAS Transactions on Applied and Theoretical Mechanics (2014).

[18] H. Bajas et al., "Test Result of the Short Models MQXFS3 and MQXFS5 for the HLLHC Upgrade," IEEE Transactions on Applied Superconductivity, vol. 28, no. 3, pp. 1-6, Art no. 4007006 (2018).

[19] M. Marchevsky, "Acoustic sensors", ASC 2020 Wk1LOr3B-06, to be published.

[20] M. Calvi, E. Floch, S. Kouzue and A. Siemko, "Improved quench localization and quench propagation velocity measurements in the LHC superconducting dipole magnets," in IEEE Transactions on Applied Superconductivity, vol. 15, no. 2, pp. 1209-1212, June 2005, doi: 10.1109/TASC.2005.849533.

[21] M. Marchevsky et al., "Magnetic Quench Antenna for MQXF Quadrupoles," in IEEE Transactions on Applied Superconductivity, vol. 27, no. 4, pp. 1-5, June 2017, Art no. 9000505, doi: 10.1109/TASC.2016.2642983.

[22] T. Strauss et al., "Quench Location in the LARP MQXFS1 Prototype", IEEE Transactions on Applied Superconductivity, vol. 28, no. 3, pp. 1-4, Art no. 4001604 (2018).

[23] J. DiMarco et al., "A Full-length Quench Antenna for MQXFA Production Series Quadrupole Magnet Testing", ASC 2020, submitted for publication.

[24] M. Marchevsky et al., "Acoustic detection in superconducting magnets for performance characterization and diagnostics", CERN Yellow Report CERN2013-006, pp.38-42.

[25] A. Devred, "Quench origins", AIP Conf.Proc. 249 (1992) 1262-1308.

[26] L. Bottura, "Magnet quench 101 ", CERN Yellow Report CERN-2013-006, pp.1-9, 2013.

[27] A. Siemko, "Magnet Quench Process", Part of LEP performance, Proceedings, 11th Workshop, Chamonix, France, January 15-19, 2001.

[28] Martin N. Wilson, Paul Seidel (Editor), "Fundamentals in Superconducting Magnets" in "Applied Superconductivity: Handbook on Devices and Applications", Vol. 2., Wiley-VCH.

[29] P. P. Granieri, C. Lorin and E. Todesco, "Slip-Stick Mechanism in Training the Superconducting Magnets in the Large Hadron Collider," in IEEE Transactions on Applied Superconductivity, vol. 21, no. 5, pp. 3555-3560, Oct. 2011, doi: 10.1109/TASC.2011.2162727.

[30] P. Ferracin et al., "Thermal, electrical and mechanical response in Nb/sub 3/Sn superconducting coils," in IEEE Transactions on Applied Superconductivity, vol. 14, no. 2, pp. 361-364, June 2004, doi: 10.1109/TASC.2004.829130.

[31] H. Bajas et al., "Quench Analysis of High-Current-Density Nb3Sn Conductors in 
Racetrack Coil Configuration“, IEEE Trans.Appl.Supercond. 25 (2015) no.3, 4004005

[32] G. Ambrosio et al., "Measurement of Inter-Strand Contact Resistance in Epoxy Impregnated Nb3Sn Rutherford Cables", AIP Conference Proceedings 711, 828, 2004.

[33] L. Bottura, M. Breschi, E. Felcini, A. Lechner, "Stability margin of the LHC Nb-Ti Magnets subjected to beam losses", Phys. Rev. Accel. Beams 22, 041002 (2019).

[34] M. Breschi, E. Felcini, L. Bottura, "Quench Level of the HL-LHC Nb3Sn IR Quadrupoles", IEEE Trans. Appl. Supercond., vol. 27, n. 4, 7782864, 2017.

[35] G. D. Brittles et al., "Persistent current joints between technological superconductors", Supercond. Sci. Technol. 28 093001, 2015.

[36] S. Delchamps, et al., "AC Loss Measurement of SSC Dipole Magnets," Proceedings of the ICFA Workshop on AC Superconductivity, Tsukuba, June 1992, p. 19.

[37] R. Soika, M. D. Anerella, A.K Ghosh, P. Wanderer, M.N. Wilson, W.V. Hassenzahl, J. Kaugerts, and G. Moritz. Interstrand resistance measurements in cored Nb-Ti Rutherford cables. IEEE Trans. Appl. Supercond., 13:2380 2383, 2003.

[38] Devred, et al., "Interstrand Resistance Measurements on Nb3Sn Rutherford-Type Cables", in IEEE Trans. Appl. Supercond., vol 9, pp 722-726, 1999.

[39] A. V. Zlobin et al., "Testing of a single $11 \mathrm{~T}$ Nb3Sn dipole coil using a dipole mirror structure," presented at the International Particle Accelera-tor Conference, Dresden, Germany, 2014, Paper WEPRI099.

[40] Daniel Schoerling and Alexander V. Zlobin (editors), "Nb3Sn Accelerator Magnets", Springer Open (2019). 